\title{
A multi-gene analysis strategy identifies metabolic pathways targeted by trans-10, cis-12-conjugated linoleic acid in the liver of hamsters
}

\author{
Virginia Navarro ${ }^{1}$, María P. Portillo ${ }^{1}$, Alain Margotat ${ }^{2,3,4}$, Jean-François Landrier ${ }^{2,3,4}$, Maria T. Macarulla ${ }^{1}$, \\ Denis Lairon ${ }^{2,3,4}$ and Jean-Charles Martin ${ }^{2,3,4 *}$ \\ ${ }^{1}$ Department of Nutrition and Food Science, Faculty of Pharmacy, University of the Basque Country, Vitoria E-01006, Spain \\ ${ }^{2}$ INRA, UMR1260 'Nutriments Lipidiques et Prévention des Maladies Métaboliques', F-13385 Marseille, France \\ ${ }^{3}$ INSERM, U476, F-13385 Marseille, France \\ ${ }^{4}$ Faculté de Médecine, Université Aix-Marseille 1, Université Aix-Marseille 2, IPHM-IFR 125, F-13385 Marseille, France
}

(Received 14 August 2008 - Revised 29 October 2008 - Accepted 24 November 2008 - First published online 16 February 2009)

In mice, hepatic functions can be greatly affected by dietary trans-10, cis-12-conjugated linoleic acid (CLA). However, this phenomenon has been less documented in hamsters. In the present study, male hamsters were fed two doses of the trans-10, cis-12-CLA (0.5 and $1 \%$, w/w diet) or linoleic acid $(0.5 \%)$ for 6 weeks. The effects on the liver were examined by measuring the expression of thirty-six genes representing key metabolic pathways. CLA-responsive genes and their relationships with physiological outcomes were examined by a multivariate analysis procedure. Compared with control hamsters, those receiving either 0.5 or $1 \%$ CLA exhibited similar fat loss $(15-24 \% ; P \leq 0 \cdot 05)$ and liver enlargement $(21-28 \% ; P \leq 0.05)$, with no signs of steatosis. We also observed a dose-dependent increase in the transcription of genes involved in lipid breakdown and lipid harvesting from blood, and in genes related to the oxidative stress and inflammatory responses. These responsive genes varied in parallel with cell membrane lipids $\left(R^{2} 0 \cdot 31-0.42\right)$ and to a lesser extent with liver enlargement $\left(R^{2} 0 \cdot 22\right)($ all $P<0 \cdot 05)$. We conclude that in hamsters, liver enlargement induced by trans-10, cis-12-CLA is accompanied by an increased metabolic potential to process fatty acids from mobilised adipose stores. This elevated metabolic activity, comprised of anabolic pathways and their catabolic counterparts, can trigger inflammation and the oxidant stress defence pathways in a dose-dependent manner. These results provide novel insights into the mechanisms by which trans-10, cis-12-CLA affects pathways related to liver function.

Conjugated linoleic acid: Liver gene expression: Hamsters: Lipid metabolism: Partial least square regression

The term conjugated linoleic acid (CLA) describes a group of linoleic acid isomers whose double bonds are not separated by a methylene group, but are conjugated. Some of these isomers are present in small amounts in foods derived from ruminant animals $^{(1)}$. In recent years, CLA has received a great deal of attention because of the multiple beneficial health-related effects reported in various animal models, especially with reference to cancer, obesity and atherosclerosis ${ }^{(23)}$. Despite these positive effects, adverse effects of CLA such as lipid peroxidation $^{(4)}$, insulin resistance ${ }^{(4-6)}$, fatty liver ${ }^{(5,7,8)}$ and oxidative stress ${ }^{(9)}$ remain a matter of concern. Particularly, many studies using the mouse as a model have reported deleterious effects on the liver associated with chronic consumption of the trans-10, cis-12-CLA isomer. These effects are usually accompanied by a dramatic loss of adipose tissue and the development of insulin resistance ${ }^{(5,7,10,11)}$. As a result, the liver is one of the main target organs in studies examining the impact of the trans-10, cis-12-CLA isomer on health outcomes.

Not all species are as CLA sensitive as the mouse with regard to liver enlargement and steatosis, and the origin of such a species-specific discrepancy is largely unknown at present $^{(7)}$. Therefore, any complete extrapolation of the mouse findings to man would be inappropriate. The body fat reduction observed in the hamster in response to dietary CLA is not as dramatic as that observed in the mouse, and this effect in the hamster seems to be intermediate between that of mice and man ${ }^{(12)}$. In addition, unlike mice, consumption of CLA in hamsters brings about a moderate liver enlargement that is not due to fat deposition ${ }^{(13,14)}$. Hence, the hamster model could make new and complementary contributions to our understanding of the effects of trans-10, cis-12-CLA on the liver.

Increased lipogenesis ${ }^{(7,15)}$, inflammation ${ }^{(16)}$, lysosomal deficiency $^{(17)}$, changes in lipid oxidation ${ }^{(7,18)}$ and oxidative stress $^{(19)}$ all stand out among the pathways in the liver that may accompany liver enlargement and be affected by trans-10, cis-12-CLA feeding. In the present study, we aimed to determine the expression levels of thirty-six representative genes of these pathways in the liver of hamsters fed a diet enriched with either linoleic acid or two doses of trans-10, cis-12-CLA $(0.5$ or $1 \%$, by weight $)$. This is the

Abbreviations: CLA, conjugated linoleic acid; PLS, partial least square.

* Corresponding author: Dr Jean-Charles Martin, fax +33 491782 101, email jean-charles.martin@univmed.fr 
first study to provide such a comprehensive gene expression pattern in the hamster liver. Gene expression profiling was carried out by the means of multivariate statistical analysis to improve interpretation of the results.

\section{Materials and methods}

\section{Animals, diets and experimental design}

The experiment, conducted with thirty male Syrian Golden hamsters aged 9 weeks and purchased from Harlan Iberica (Barcelona, Spain), took place in accordance with Spanish guidelines for the use and care of laboratory animals and was approved by the Ethical Committee of the University of País Vasco. The animals were individually housed in polycarbonate metabolism cages (Techniplast Gazzada, Guguggiate, Italy) and placed in an air-conditioned room $\left(22 \pm 2{ }^{\circ} \mathrm{C}\right)$ with a $12 \mathrm{~h}$ light-dark cycle. The experiments were initiated after a $6 \mathrm{~d}$ adaptation period.

After the $6 \mathrm{~d}$ adaptation period, hamsters were randomly divided into three dietary groups of ten animal each, and each group was fed a designated dose of trans-10, cis-12CLA as NEFA $(0,0.5$ and $1.0 \mathrm{~g} / 100 \mathrm{~g}$ diet $)$ in a semi-purified atherogenic diet. The diet consisted of (per $\mathrm{kg}$ ): $200 \mathrm{~g}$ casein (Sigma, St Louis, MO, USA); $4 \mathrm{~g}$ L-methionine (Sigma); $200 \mathrm{~g}$ wheat starch (Vencasser, Bilbao, Spain); $405 \mathrm{~g}$ sucrose (local market); $100 \mathrm{~g}$ palm oil (Agra-Unilever, Leioa, Spain); $30 \mathrm{~g}$ cellulose (Vencasser), $4 \mathrm{~g}$ choline-HCl (Sigma); $1 \mathrm{~g}$ cholesterol (Sigma). Trans-10, cis-12-CLA (95\%) was supplied by Natural Lipids Ltd (Hovdebygda, Norway). Vitamin $(11 \mathrm{~g} / \mathrm{kg})$ and mineral $(40 \mathrm{~g} / \mathrm{kg})$ mixes were formulated according to AIN-93 guidelines and supplied by ICN Pharmaceuticals (Costa Mesa, CA, USA). The experimental diets were freshly prepared once per week, gassed with $\mathrm{N}_{2}$, and stored at $0-4^{\circ} \mathrm{C}$ to avoid rancidity. All animals had free access to food and water and were killed in the middle of the dark period.

\section{Sampling}

At the end of the experimental period, blood samples were collected under inhalation anaesthesia (diethyl ether) by cardiac puncture. Serum was obtained by centrifugation (1000 $g$ for $10 \mathrm{~min}$ at $4^{\circ} \mathrm{C}$ ) and stored at $-80^{\circ} \mathrm{C}$ until analysis.

Adipose tissue from different anatomical regions (perirenal, epididymal and gluteal subcutaneous) and liver were dissected and weighed.

\section{Serum analysis}

Serum cholesterol, TAG and glucose levels were measured by spectrophotometry using commercial kits (BioSystems, Barcelona, Spain). Insulin levels were measured by RIA (Linco, St Charles, MO, USA).

\section{Liver analysis}

Total lipids were extracted from the liver following the method described by Folch et al. ${ }^{(20)}$. The lipid extract was dissolved in isopropanol. Total, non-esterified and esterified cholesterol was determined as previously described ${ }^{(14)}$, and phospholipids and TAG were measured by spectrophotometry using a commercial kit (BioSystems, Barcelona, Spain).

Liver glycogen was analysed spectrophotometrically by the method described ${ }^{(21)}$.

Extraction and analysis of RNA from liver and quantification by real-time polymerase chain reaction

Total RNA was extracted from the livers of the hamsters using Trizol, according to the manufacturer's instructions. The RNA concentration was determined from the absorbance at $260 \mathrm{~nm}$. A quantity of $1.5 \mu \mathrm{g}$ of total RNA from each sample was reverse-transcribed to cDNA in a final volume of $30 \mu \mathrm{l}$ by using an iScript ${ }^{\mathrm{TH}}$ cDNA synthesis kit (BioRad).

Gene expression was determined by real-time PCR (Stratagene Mx 3005; La Jolla, CA, USA) using the SYBR Green method ${ }^{(22)}$, quantified using $18 \mathrm{~S}$ as the reference gene, and expressed as mean normalised expression (MNE) using the qgene application ${ }^{(23)}$. The formula MNE = $\mathrm{E}_{\mathrm{ref}}^{\mathrm{CTref}}$, mean $/ \mathrm{E}_{\text {target }}^{\mathrm{CT} \text { Ttarget, mean }}$ (where $\mathrm{E}$ is PCR efficiency, CT mean is cycle threshold mean of duplicate or triplicate) was used to transform the cycle threshold unit into a linear unit of normalised expressions. The choice of the oligonucleotide sequences was based on available hamster data, or on the most conserved sequences among mice, and/or rat and/or human aligned sequences when hamster data were missing (see supplementary on-line material Table 1). Primer design was performed with Primer express (Applied Biosystems, Foster City, CA, USA) for quantitative RT-PCR, whenever necessary. Basic Local Alignment Search Tool (BLAST) analysis indicated that there was no similarity with other known hamster sequences when other species were used to obtain primers for unpublished hamster gene sequences.

\section{Statistical analysis}

From each group of ten hamsters, we obtained a complete set of results for seven to eight hamsters. Although partial least square (PLS) statistical methods (see explanation below) can handle missing data, hamsters with too many missing genes (expression of over three genes missing per hamster) were discarded. Results are presented as mean values and standard deviations in Tables. Statistical analysis of physiological parameters and gene expression data was performed by one-way ANOVA followed by the Fisher post hoc test, using Statview (SAS Institute, Inc., Cary, NC, USA). Statistical significance was set with a $P$ level at $0 \cdot 05$ (see on-line supplemental Fig. 1).

Moreover, an additional multifactorial statistical analysis that took into account the effects of the trans-10, cis-12CLA on all the genes together was performed. This statistical procedure allowed us to linearly reduce the gene expression profile of each hamster (thirty-six genes or variables) down to one score (one variable) (Fig. 1). We then examined how these scores could predict hamsters' class membership (Y response, for example, dietary treatment), or predict phenotypic outcomes (biochemical assays or tissue weights as $\mathrm{Y}$ responses). From this analysis, it was possible to retrospectively determine which of the original RT-PCR variables (gene expression values) had prediction values over all genes that were most consistent with $\mathrm{Y}$. This approach allowed us to identify CLA target genes (Fig. 1). 
Table 1. Tissue weights, liver composition and serum parameters in hamsters fed on the experimental diets for 6 weeks

(Mean values and standard deviations for eight to ten hamsters per group)

\begin{tabular}{|c|c|c|c|c|c|c|}
\hline \multirow[b]{2}{*}{ Physiological parameters } & \multicolumn{2}{|c|}{ Linoleic acid } & \multicolumn{2}{|c|}{ CLA $0.5 \%$} & \multicolumn{2}{|c|}{ CLA $1 \%$} \\
\hline & Mean & SD & Mean & SD & Mean & SD \\
\hline Adipose tissue $(\mathrm{g})^{\star}$ & $6 \cdot 2^{b}$ & 0.9 & $5 \cdot 2^{a}$ & 0.8 & $5 \cdot 0^{\mathrm{a}}$ & 0.7 \\
\hline Liver (g) & $6 \cdot 3^{a}$ & 0.6 & $7 \cdot 3^{\mathrm{b}}$ & 0.7 & $7 \cdot 3^{\mathrm{b}}$ & 1.2 \\
\hline \multicolumn{7}{|l|}{ Liver glycogen } \\
\hline $\mathrm{mg} / \mathrm{g}$ & 54 & $8 \cdot 3$ & 52 & $10 \cdot 2$ & 54 & 4.9 \\
\hline mg/liver & $341^{\mathrm{a}}$ & 65 & $393^{\mathrm{a}}$ & 87 & $422^{b}$ & 47 \\
\hline \multicolumn{7}{|l|}{ Liver TAG } \\
\hline $\mathrm{mg} / \mathrm{g}$ & $5 \cdot 0^{\mathrm{b}}$ & 0.9 & $4 \cdot 1^{\mathrm{a}}$ & 0.6 & $5 \cdot 5^{\mathrm{b}}$ & 0.8 \\
\hline $\mathrm{mg} / \mathrm{liver}$ & $30 \cdot 9^{\mathrm{a}}$ & 5.6 & $29 \cdot 2^{\mathrm{a}}$ & $6 \cdot 0$ & $40 \cdot 1^{\mathrm{b}}$ & 8.1 \\
\hline \multicolumn{7}{|c|}{ Liver non-esterified cholesterol } \\
\hline $\mathrm{mg} / \mathrm{g}$ & $1.4^{\mathrm{a}}$ & 0.13 & $1.8^{\mathrm{b}}$ & 0.3 & $1.9^{\mathrm{b}}$ & 0.2 \\
\hline $\mathrm{mg} /$ liver & $9 \cdot 0^{\mathrm{a}}$ & 1.0 & $12 \cdot 9^{\mathrm{b}}$ & $2 \cdot 7$ & $13 \cdot 5^{\mathrm{b}}$ & 2.5 \\
\hline \multicolumn{7}{|l|}{ Liver phospholipids } \\
\hline $\mathrm{mg} / \mathrm{g}$ & $11 \cdot 7^{\mathrm{a}}$ & 0.8 & $12 \cdot 6^{\mathrm{a}}$ & $2 \cdot 1$ & $13 \cdot 6^{\mathrm{b}}$ & 1.9 \\
\hline mg/liver & $73 \cdot 6^{\mathrm{a}}$ & 7.5 & $92 \cdot 5^{\mathrm{b}}$ & $21 \cdot 7$ & $97 \cdot 6^{\mathrm{b}}$ & $13 \cdot 3$ \\
\hline \multicolumn{7}{|l|}{ Liver cholesteryl ester } \\
\hline $\mathrm{mg} / \mathrm{g}$ & $5 \cdot 6^{\mathrm{b}}$ & 0.7 & $4 \cdot 1^{a}$ & 0.8 & $4.5^{\mathrm{a}}$ & 0.5 \\
\hline $\mathrm{mg} / \mathrm{liver}$ & $35 \cdot 2$ & $6 \cdot 2$ & $30 \cdot 2$ & $7 \cdot 8$ & 33 & $6 \cdot 1$ \\
\hline Plasma TAG (mmol/l) & 3.1 & 1.1 & 2.4 & 0.8 & $2 \cdot 7$ & 0.8 \\
\hline Plasma glucose $(\mathrm{mmol} / \mathrm{l})$ & 8.0 & 1.8 & 7.5 & 1.8 & $7 \cdot 2$ & 1.4 \\
\hline Insulin $(\mu \mathrm{g} / \mathrm{l})$ & 3.4 & $2 \cdot 0$ & 3.7 & 1.6 & 3.7 & 0.8 \\
\hline
\end{tabular}

CLA, conjugated linoleic acid.

${ }^{\mathrm{a}, \mathrm{b}}$ Mean values within a row with unlike superscript letters were significantly different $(P<0.05$; one-way ANOVA).

${ }^{*}$ Adipose tissue mass refers to the sum of epididymal, perirenal and gluteal subcutaneous fat depots.

The concept of megavariate statistics for biological purposes is also depicted in simple concepts by Grainger (DJ Grainger, personal communication; http://www.graingerlab. org/Research/Minireviews/reviews.html), and detailed in Trygg et al. ${ }^{(24)}$. A similar approach was applied to study the CLA effects on the liver proteome of mice ${ }^{(25)}$. In this approach, all genes were considered collectively with regard to gene expression response to dietary CLA, and not separately, as in ANOVA analysis. The two methods can give rise to some dissimiliarities (see on-line supplemental Fig. 1), but the multivariate analysis appears more appropriate when addressing a multicomponent biological response.

Multivariate statistics were performed using SIMCA P-11 (Umetrix, Umea, Sweden), after centring and unit variance

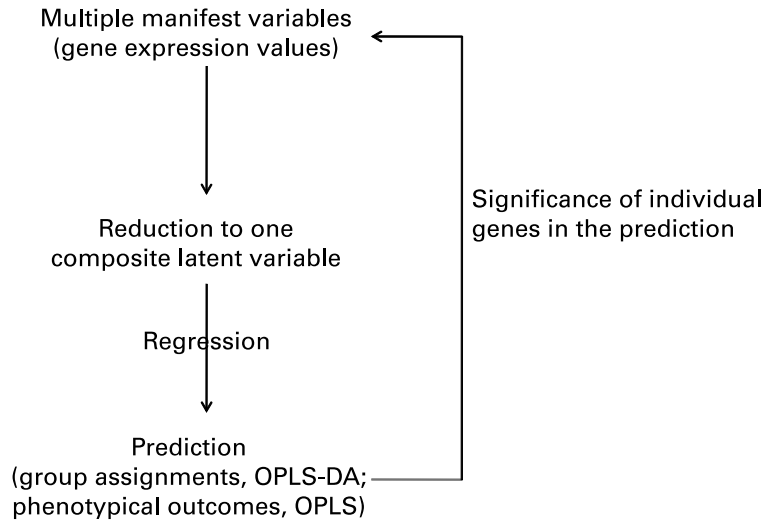

Fig. 1. Outline of the multidimensional statistical analysis. OPLS-DA, orthogonal partial least square-discriminant analysis. scaling of the data. The characterisation of the effects of CLA on the gene expression pattern was examined by using a projection on latent structure discriminant analysis (PLS-DA), with diet as the class determinant. In addition, we examined the relationships between gene expression (X variables) and phenotypic outcome ( $\mathrm{Y}$ variables) using PLS regression. These methods are principal component based-methods. We specifically used the orthogonal PLS-DA and orthogonal PLS procedures, which allow for filtering out of the variations among the $\mathrm{X}$ variables that are not correlated to $\mathrm{Y}$, thus making the orthogonally treated data more precise and easier to interpret ${ }^{(26)}$. The significant relevance of the variables of interest was determined by jack-knifing ${ }^{(27)}$, using $99 \% \mathrm{CI}$.

The relationships between genes targeted by CLA were then examined by clustering methods, using permutmatrix (www.lirmm.fr/ $\sim$ caraux/PermutMatrix/) ${ }^{(28)}$, with the Ward method as clustering conditions, and using Euclidian distances. Values were calculated relative to the linoleic acid control hamster values, and $\log _{2}$-transformed to offset skewed distribution.

\section{Results}

\section{Physiological parameters}

No differences in food intake (6.0 (SD $0 \cdot 1) \mathrm{g} / \mathrm{d}$ in the control group, $5.7(\mathrm{SD} 0.1) \mathrm{g} / \mathrm{d}$ in the $0.5 \%$ trans- 10 , cis-12-CLA group and 5.7 (SD 0.1) $\mathrm{g} / \mathrm{d}$ in the $1.0 \%$ trans-10, cis-12CLA group) were observed among the three experimental groups. Although no significant differences in final body weight were found between hamsters fed the CLA diets and 
those fed the control diet (121 (SD 3) $\mathrm{g}$ in the control group, 119 (SD 1) $\mathrm{g}$ in the $0.5 \%$ trans-10, cis-12-CLA group and 124 (SD 2) $g$ in the $1.0 \%$ trans-10, cis-12-CLA group), hamsters fed the $1 \%$ CLA diet showed significantly greater final body weight than those fed the $0.5 \%$ CLA diet.

Data describing tissue weights, liver composition and serum parameters are shown in Table 1. Both doses of trans-10, cis-12-CLA led to a significant reduction in adipose fat pad mass and a significant increase in liver size $(P<0.05)$. With regard to liver lipids, both CLA doses increased non-esterified cholesterol $(P<0 \cdot 01)$, whereas only the highest dose significantly increased phospholipids. Both doses decreased the cholesteryl ester concentration $(P<0 \cdot 01)$ in liver lipid stores, but the low dose only reduced TAG content $(P<0 \cdot 05)$. By contrast, no changes in liver glycogen content were observed in CLA-fed groups. CLA feeding did not modify serum parameters.

\section{Gene expression analysis}

We selected thirty-six genes in the liver that could be targeted by trans-10, cis-12-CLA feeding and that cover a wide range of biological function (Fig. 2). Two were not significantly detected in the liver (cyclo-oxygenase- 2 and PPAR $\gamma$ ), and this was not due to a primer failure, as indicated by a validation of the assay.

Discriminant analysis showed a significant shift of gene expression upon CLA feeding, allowing for clear group assignment (Fig. 3 and Fig. 4 (a)). From this model we determined which genes were preferentially targeted at 0.5 or $1 \%$ dietary CLA over all genes, by reference to control linoleic acid-fed hamsters. The main responsive genes after CLA feeding were thus identified among the thirty-six genes analysed. The results are displayed as a heat map showing the association (PLS regression values) between gene expression and selected outcomes (Figs. 4 (a), (b) and (c)). The statistical levels were defined by jack-knifing at $99 \%$ of CI. In this situation, twelve genes were especially important for differentiation between the $0.5 \%$ CLA treatment and the control diet (Fig. 4 (a)). Twelve genes were additionally up-regulated when the amount of the trans-10, cis-12-CLA given to hamsters was raised to $1 \%$. Thus, the most significant gene activation occurred at the $1 \%$ dose, including genes involved in lipid transport, catabolism and synthesis, together with markers of inflammation and oxidative stress (Fig. 4 (a)).

The phenotypic outcomes of the genes with modified expression at the 0.5 and the $1 \%$ trans-10, cis-12-CLA

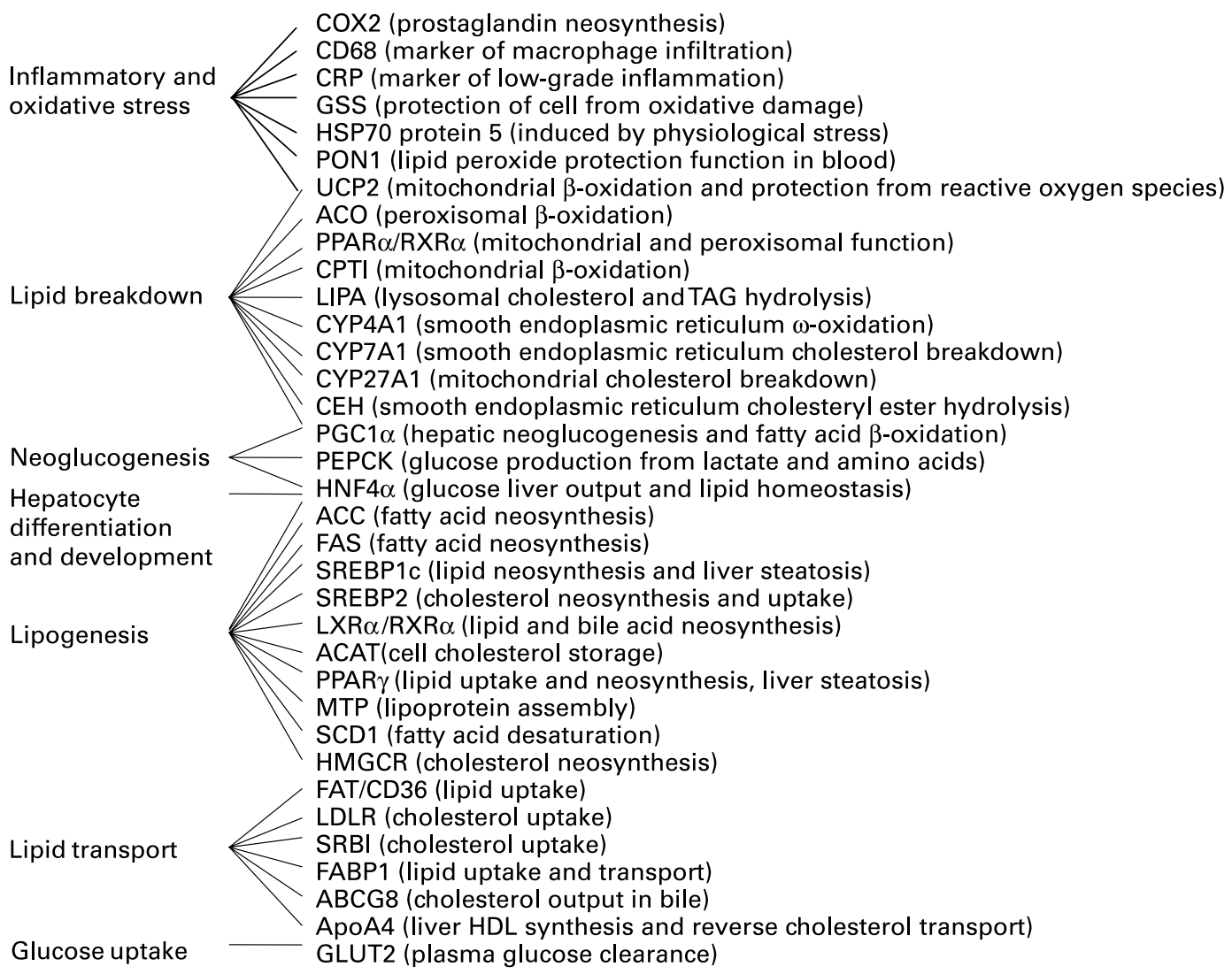

Fig. 2. Gene names and functions, as well as their expression levels in the liver, as determined by real-time PCR. COX2, cyclo-oxygenase 2; CD68, cluster of differentiation 68; CRP, C-reactive protein; GSS, glutathione S-transferase; HSP70 protein 5, heat-shock protein-70 kDa-protein 5; PON1, paraoxonase-1; UCP2, uncoupling protein 2; ACO, acyl CoA oxidase; RXR $\alpha$, retinoic acid X receptor $\alpha$; CPTI, carnitine palmitoyl-transferase I; LIPA, acid lipase; CYP4A1, cytochrome P450 4A1; CYP7A1, cytochrome P450 7A1; CYP27A1, cytochrome P450 27A1; CEH, cholesteryl ester hydrolase; PGC1 $\alpha$, PPAR $\gamma$ coactivator $1 \alpha$; PEPCK, phosphoenolpyruvate carboxykinase; HNF4 $\alpha$, hepatocyte nuclear factor $4 \alpha$; ACC, acetyl CoA carboxylase; FAS, fatty acid synthase; SREBP1c, sterol regulatory element binding protein 1c; SREBP2, sterol regulatory element binding protein 2; LXR $\alpha$, liver X receptor $\alpha$; ACAT, acylcholesterol acyltransferase; MTP, microsomal transfer protein; SCD1, stearoyl CoA desaturase 1; HMGCR, hydroxymethyl-glutaryl CoA reductase; FAT/CD36, fatty acid transporter/cluster of differentiation 36; LDLR, LDL receptor; SRBI, scavenger receptor type BI; FABP1, fatty acid binding protein 1; ABCG8, ATP binding cassette G8; apoA4, apoprotein A4. 
(a)

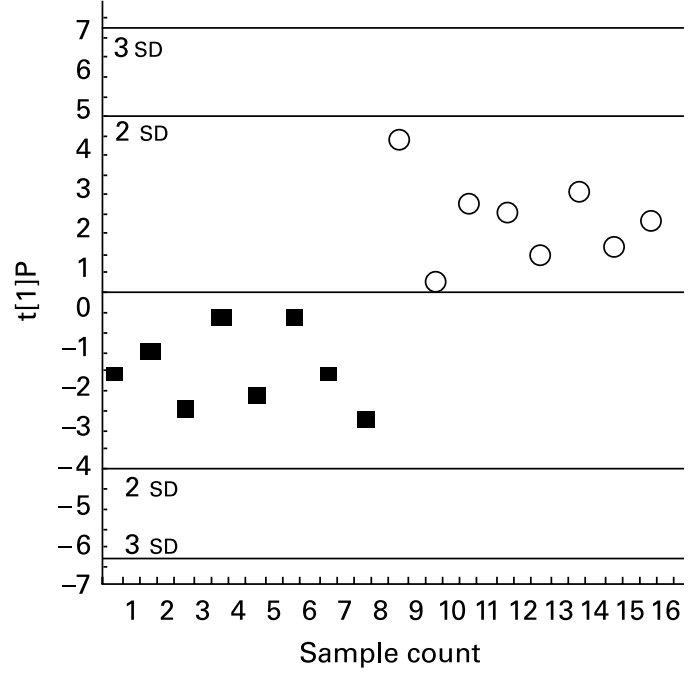

(b)

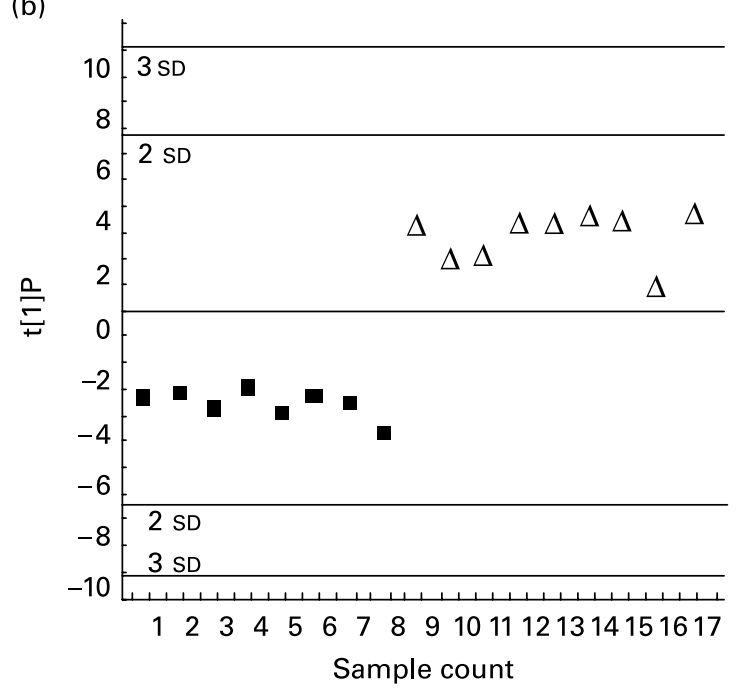

Fig. 3. Orthogonal projection on latent structure-discriminant analysis of the genes expressed in the liver of hamsters given either $0.5 \%$ of linoleic acid (control; $\mathbf{\square}$ ), $0.5 \%$ of trans-10, cis-12-conjugated linoleic acid (CLA) (O) or $1 \%$ of trans-10, cis-12-CLA $(\Delta)$ (by weight). The score plots display the complete separation of the hamsters fed with $0.5 \%(a)$, or $1 \%$ CLA (b), from those fed with linoleic acid. Plots are based on overall gene expression profiles. t[1]P, multigeric score value for individual hamsters.

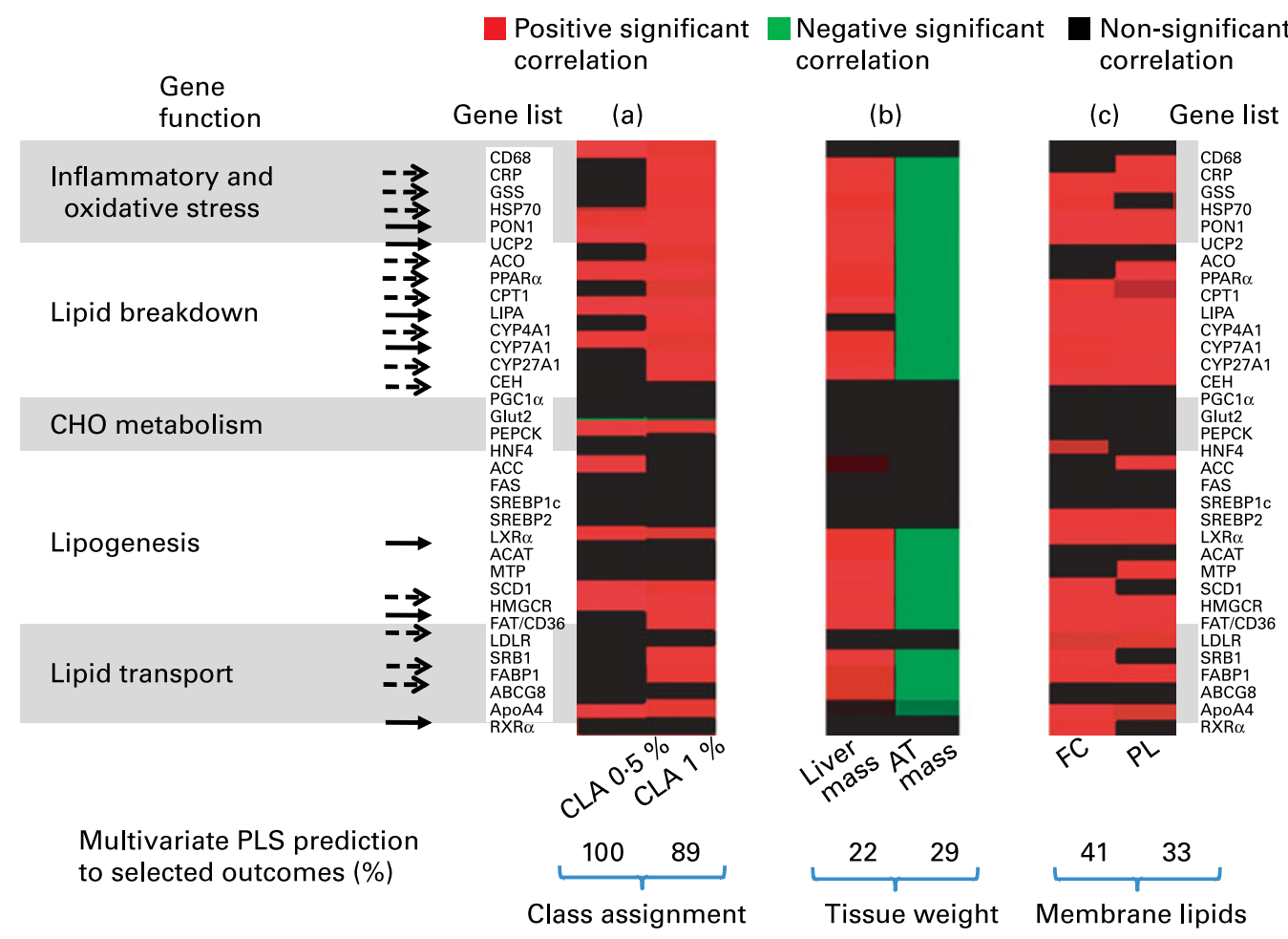

Fig. 4. Heat map displaying the statistical significance of the partial least square (PLS) regression coefficients among individual gene expression and selected outcomes. Significant coefficients (determined by jack-knifing with $99 \% \mathrm{Cl}$ ) are represented as red boxes (for positive correlation), green boxes (for negative correlation) or black boxes (NS). Colour intensity indicates lower (dark) to higher (bright) correlation coefficients. (a) PLS regression coefficients among gene expression and class membership, for example, hamsters fed with 0.5 or $1 \%$ conjugated linoleic acid (CLA) (groups built as dummy dependent variables). (b) PLS regression coefficients among gene expression and tissue masses. (c) PLS regression coefficients among liver membrane lipids. Genes are clustered according to biological functions. Arrows indicate the CLA-responsive genes associated with the observed phenotypic outcomes (-- > genes activated at the $0.5 \%$ CLA dose; $[\rightarrow$ ], genes activated at both the 0.5 and $1 \%$ CLA dose). There were eight to nine hamsters per group. CD68, cluster of differentiation $68 ;$ CRP, C-reactive protein; GSS, glutathione S-transferase; HSP70, heat-shock protein-70 kDa-protein 5; PON1, paraoxonase-1; UCP2, uncoupling protein 2; ACO, acyl CoA oxidase; CPTI, carnitine palmitoyl-transferase I; LIPA, acid lipase; CYP4A1, cytochrome P450 4A1; CYP7A1, cytochrome P450 7A1; CYP27A1, cytochrome P450 27A1; CEH, cholesteryl ester hydrolase; PEPCK, phosphoenolpyruvate carboxykinase; HNF4, hepatocyte nuclear factor 4; ACC, acetyl CoA carboxylase; FAS, fatty acid synthase; SREBP1c, sterol regulatory element binding protein 1c; SREBP2, sterol regulatory element binding protein 2; LXR $\alpha$, liver X receptor $\alpha$; ACAT, acylcholesterol acyltransferase; MTP, microsomal transfer protein; SCD1, stearoyl CoA desaturase 1; HMGCR, hydroxymethyl-glutaryl CoA reductase; FAT/CD36, fatty acid transporter/cluster of differentiation 36; LDLR, LDL receptor; SRBI, scavenger receptor type Bl; FABP1, fatty acid binding protein 1; ABCG8, ATP binding cassette G8; ApoA4, apoprotein A4; RXR $\alpha$, retinoic acid X receptor $\alpha$; CHO, carbohydrate; AT, adipose tissue; FC, non-esterified cholesterol; PL, phospholipids. 
doses were then examined by the means of multivariate PLS regression models. The multivariate gene expression analysis indicated that $22 \%$ of both the liver mass changes and of the adipose fat pad mass were associated with the CLAinduced gene expression in the liver $(P<0 \cdot 02)$ (Fig. 4 (b)). Interestingly, the same set of genes that were up-regulated by CLA feeding and that were associated with increased liver mass also corresponded to a decrease of adipose fat mass (Fig. 4 (b)). Specifically, nine out of the twelve genes up-regulated at $0.5 \%$ CLA, and eighteen out of the twentyfour genes up-regulated at $1 \%$ CLA, were associated with changes in liver mass and/or adipose fat pad masses.

We also found some positive relationships between the gene expression pattern and liver membrane lipids, namely nonesterified cholesterol and phospholipids (multivariate prediction coefficient $R^{2} 0.41, P=0.0018$ and $R^{2} 0.32, P=0.0082$ with non-esterified cholesterol and phospholipids, respectively, with 0.5 and $1 \%$ CLA), but not with liver cytosolic stored lipids (non-significant statistical model prediction with TAG and cholesteryl esters) (Fig. 4 (c)). Overall, these genes were related to the oxidant stress defence and to lipid metabolism (breakdown, synthesis and transport). Interestingly, none of the genes involved in carbohydrate metabolism were related to any of the phenotypic outcomes observed.

Pathways of co-regulation were further identified by performing a hierarchical clustering analysis of CLA-targeted genes. This analysis revealed three main gene clusters. One of these was most specifically targeted at the lower CLA dose, and the two others mostly corresponded to the higher dose. These clusters indicate that genes related to lipid uptake, and/or lipid synthesis and lipid breakdown, and the oxidative stress defence pathways were co-expressed (Fig. 5).

\section{Discussion}

The liver is a target organ in the study of the impact of CLA, and especially in the study of the effect of the trans-10, cis-12CLA isomer on health. Many studies carried out in the mouse model have reported deleterious effects on liver, resulting from the consumption of this isomer. These effects are usually accompanied by a dramatic loss of adipose tissue and the development of hyperinsulinaemia and insulin resistance ${ }^{(5,7)}$. Consumption of CLA in hamsters brings about a less severe liver enlargement than is observed in mice, which is not due to steatosis ${ }^{(13,14)}$. Thus, we studied this species to obtain additional information on the biochemical pathways targeted by the trans-10, cis-12-CLA. Since variation in a given gene does not usually affect a biological process on its own, we analysed the variation of thirty-six genes representative of key metabolic pathways together, in order to obtain a wide display of the possible biological consequences of CLA intake, and to look for dominant effects over minor ones. We primarily used a probabilistic approach, identifying gene expression patterns that were modified by CLA consumption, and assigning this pattern to phenotypic outcomes. Such a wide gene screening of potent biochemical pathways targeted by CLA and influencing hepatomegaly has never been performed in this model.

Our statistical models (PLS regression) predicted that $22-42 \%$ of the overall variation in tissue weight changes

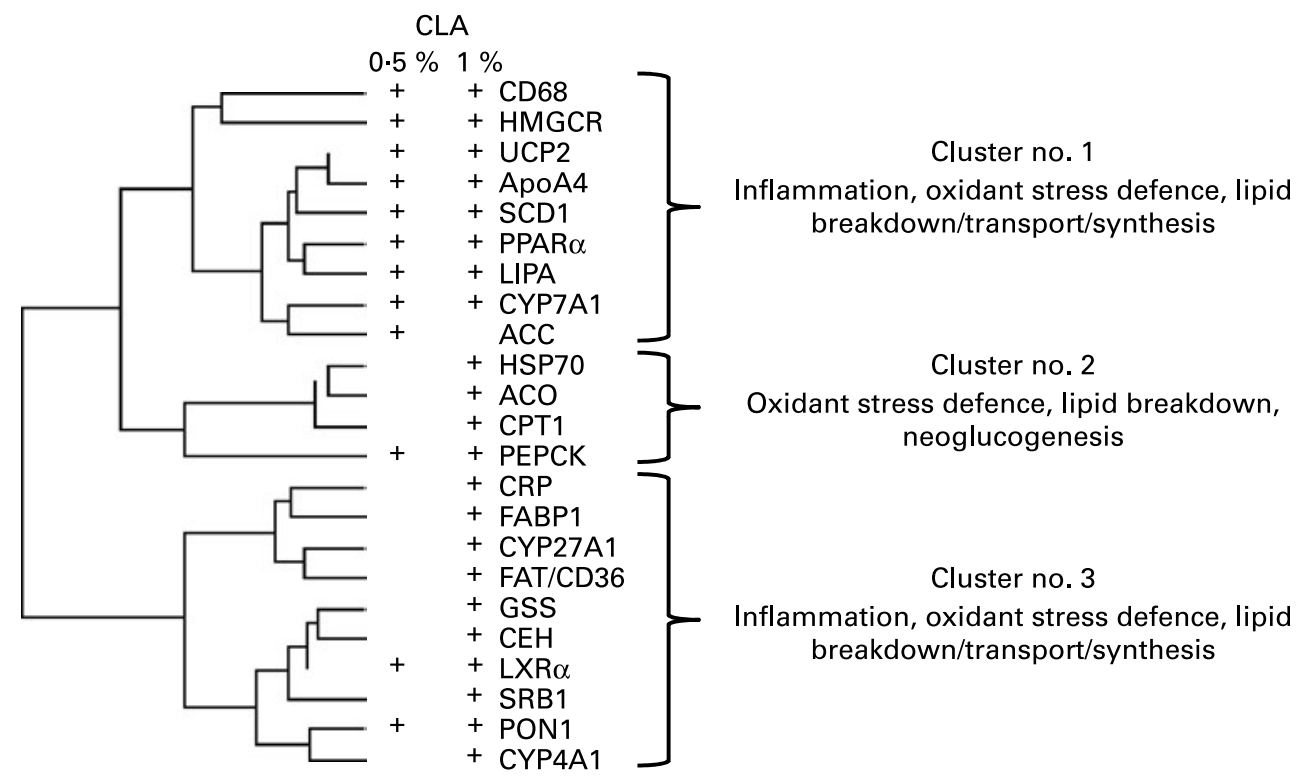

Fig. 5. Hierarchical cluster analysis of the conjugated linoleic acid (CLA)-responsive genes. Calculation was made using the clustering conditions of Ward and Euclidian distances. Data for clustering analysis were calculated from the ratio of gene expression found in linoleic acid-fed hamsters over that in CLA-fed hamsters, and after $\log _{2}$ transformation. Three main clusters can be calculated, in which the physiological functions of the clustered genes are indicated. There were twenty-five hamsters. Genes responsive to either dose as determined in Fig. 3 (a) are shown (+). CD68, cluster of differentiation 68; HMGCR, hydroxymethyl-glutaryl CoA reductase; UCP2, uncoupling protein 2; ApoA4, apoprotein A4; SCD1, stearoyl CoA desaturase 1; LIPA, acid lipase; CYP7A1, cytochrome P450 7A1; ACC, acetyl CoA carboxylase; HSP70, heat-shock protein-70 kDa-protein 5; ACO, acyl CoA oxidase; CPTI, carnitine palmitoyl-transferase I; PEPCK, phosphoenolpyruvate carboxykinase; CRP, C-reactive protein; FABP1, fatty acid binding protein 1; CYP27A1, cytochrome P450 27A1; FAT/CD36, fatty acid transporter/cluster of differentiation 36; GSS, glutathione S-transferase; $\mathrm{CEH}$, cholesteryl ester hydrolase; LXR $\alpha$, liver X receptor $\alpha$; SRBI, scavenger receptor type BI; PON1, paraoxonase-1; CYP4A1, cytochrome P450 4A1. 
or liver biochemical changes were due to CLA feeding. This relative weakness could partly arise from the time-displacement and time-scale that exist between gene, protein, metabolic and physiological events, and the sampling points. These influences may hamper correlation studies ${ }^{(29)}$. Retrospectively, it is likely that several sampling times would have improved the prediction.

Compared with linoleic acid, feeding the two doses of trans-10, cis-12-CLA produced the same physiological effect: a reduction in adipose tissue mass (15-24\%) with a commensurate liver enlargement (21-28\%). Unlike what has been observed in mice, this was not followed by dramatic liver steatosis and hyperinsulinaemia ${ }^{(7)}$. In good accordance with the present study, we and others previously found that liver enlargement in hamsters fed with $0.5 \%$ trans-10, cis-12-CLA was due to hyperplasia (published in Macarulla et al. ${ }^{(14)}$ for the hamsters of the present study), with no signs of steatosis ${ }^{(13,14)}$. Indeed, one of the main features that leads to hepatic steatosis in mice fed trans-10, cis-12-CLA is the dramatic increase in hepatic PPAR $\gamma$ and sterol regulatory element binding protein 1c (SREBP1c) due to hyperinsulinaemia, and to the fatty acid synthase gene $\mathrm{FAS}^{(5)}$. None of this took place in the present study, as insulin and SREBP1c and FAS gene expression remained unaffected, and PPAR $\gamma$ was undetectable. An additional difference can be accounted for by the efficient lipid oxidation in the hamster, which is impaired in the mouse liver through malonyl-CoA inhibition of the mitochondrial $\beta$-oxidation ${ }^{(30)}$. Nevertheless, we did not directly address this inhibition pathway in the present study.

We observed a marker of liver infiltration by potent inflammatory Kupffer cells upon CLA feeding (increase in CD68 gene expression). Such infiltration does not occur in CLAfed mice (Liu et al. ${ }^{(11)}$ ). In other situations where this takes place in the mouse model, it has been implicated in liver enlargement and steatosis ${ }^{(17)}$. This was not the case in the present study on hamsters. Another marker of inflammation, C-reactive protein, was found associated with liver enlargement. This effect could occur through CLA-induced adipose tissue inflammation $^{(31,32)}$ and may be related to observations of higher circulating C-reactive protein levels in human subjects given the trans-10, cis-12-CLA $^{(33,34)}$.

Importantly, the gene activation in the liver that we observed in response to CLA feeding was not only associated with steatosis-free hepatomegaly and adipose fat pad loss, but also with non-esterified cholesterol and phospholipids, which are primarily cell membrane components. This latter observation suggests that the increase in gene expression specifically related to lipid uptake and lipid synthesis may in part participate in the building of new membranes. Lipid degradation (fatty acid oxidation, cholesterol breakdown) would also help in preventing cytosolic accumulation and steatosis. This hypothesis is consistent with the hyperplasia observed in the hamsters of the present study ${ }^{(14)}$, thus resulting in liver enlargement. We predict that liver enlargement would increase the organ's total metabolic capacity, potentially

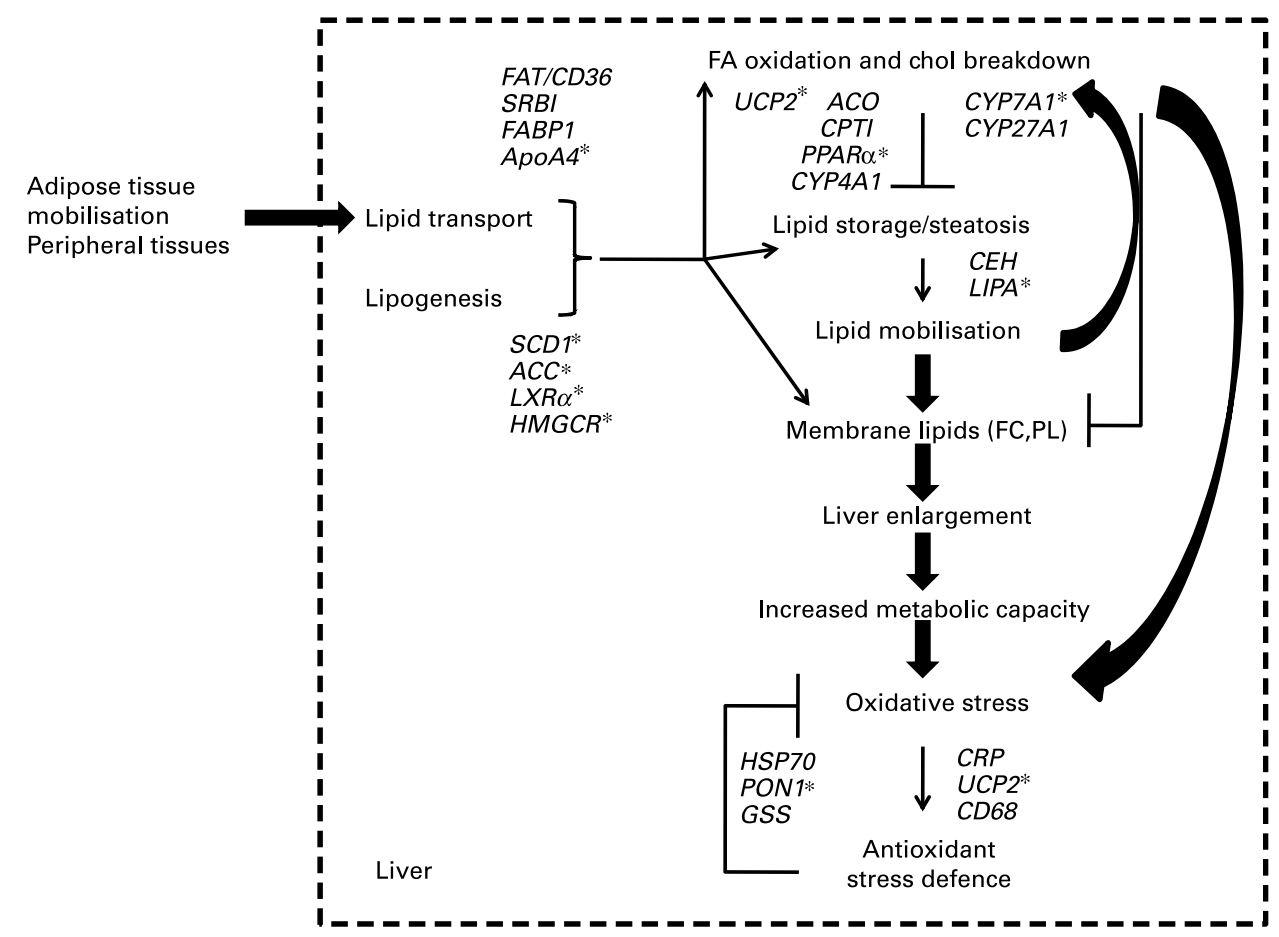

Fig. 6. Summary of the conjugated linoleic acid (CLA) effects on liver outcomes at both doses (0.5 and $1 \%)$ of the trans-10, cis-12-CLA isomer, integrated with associated gene activation. * Genes activated from the lower CLA dose (0.5\%). There were twenty-five observations (hamsters) and twenty-three variables (liver gene expression). FA, fatty acid; chol, cholesterol; FAT/CD36, fatty acid transporter/cluster of differentiation 36; SRBI, scavenger receptor type BI; FABP1, fatty acid binding protein 1; ApoA4, apoprotein A4; SCD1, stearoyl CoA desaturase 1; ACC, acetyl CoA carboxylase; LXR $\alpha$, liver X receptor $\alpha$; HMGCR, hydroxymethyl-glutaryl CoA reductase; UCP2, uncoupling protein 2; ACO, acyl CoA oxidase; CPTI, carnitine palmitoyl-transferase I; CYP4A1, cytochrome P450 4A1; CYP7A1, cytochrome P450 7A1; CYP27A1, cytochrome P450 27A1; CEH, cholesteryl ester hydrolase; LIPA, acid lipase; FC, non-esterified cholesterol; PL, phospholipids; HSP70, heat-shock protein-70 kDa-protein 5; PON1, paraoxonase-1; GSS, glutathione S-transferase; CRP, C-reactive protein; UCP2, uncoupling protein 2; CD68, cluster of differentiation 68. 
allowing it to process the fatty acids derived from the mobilised adipose stores. Doubling the trans-10, cis-12-CLA from 0.5 to $1 \%$ seemed to raise the metabolic rate to a higher level. This is indicated by the number of genes activated and involved in lipid synthesis and breakdown. This number was dramatically increased when the diet was increased from 0.5 to $1 \%$ CLA. In turn, an increase in liver metabolic activity has been suggested to produce reactive oxygen species. This results in the activation of genes involved in the oxidant stress defence as an adaptative response ${ }^{(35,36)}$. This was also observed more prominently at the $1 \%$ CLA dose (paraoxonase-1, heat-shock protein$70 \mathrm{kDa}$-protein 5, glutathione S-transferase, uncoupling protein 2) in the present study. It should be also emphasised that part of the inflammatory/antioxidant stress defence induction in the liver could also be the result of release of pro-inflammatory adipokines, as observed both in vitro ${ }^{(37)}$ and in vivo ${ }^{(32)}$. This has nevertheless not been addressed in the hamsters fed with the trans-10, cis-12-CLA.

Interestingly, the increased potential activity of the reverse cholesterol pathway that we observed at the gene expression level (increased in apoA4, and SR-BI gene expression), may participate in the improvement of atherogenic status that has been reported in hamsters fed with CLA preparations containing trans-10, cis-12-CLA $^{(38-40)}$.

The present results thus provide genomic evidence of the concerted regulation of metabolic pathways in hamsters that were chronically exposed to dietary trans-10, cis-12-CLA. An overview of the present results is summarised in Fig. 6, integrating the changes that can account for the trans-10, cis-12-CLA effects observed in our hamster model at the gene transcription level, and the biochemical and tissue levels. Particularly, trans-10, cis-12-CLA induced a liver enlargement that could improve the metabolic capacities of this organ, in order to process the fatty acids from the mobilised adipose stores. In turn, this overall increase of metabolic activity could trigger inflammation and the oxidant stress defence pathways mainly at the $1 \%$ dose, without improvement in fat loss observed from the $0.5 \%$ CLA dose. The multidimensional statistical approach developed here has opened novel perspectives for understanding the mechanisms by which trans-10, cis-12-CLA could affect pathways related to liver functions. Targeted mechanistic studies are nevertheless required to confirm our findings and hypothesis.

Palm oil was a generous gift from Agra-Unilever Foods España S.A. (Leioa, Spain).

\section{Acknowledgements}

The present study was supported by grants from the Ministerio de Ciencia y Tecnología (BFI2002-00 273) and the University of País Vasco (00 101.125-15340/2003). V. N. has a doctoral fellowship from the Spanish Government (Ministerio de Educación y Ciencia). Part of the experiment was funded by the Institut National de la Recherche Agronomique (INRA) and by the Institut National de la Recherche Médicale (INSERM), France.

M. P. P. and M. T. M. designed the study and contributed to the drafting of the paper. J.-F. L. and A. M. designed the primer database and contributed to the drafting of the paper.
D. L. contributed to the drafting of the paper. V. N. did the experimental work, designed the primer database and contributed to the drafting of the paper. J.-C. M. contributed to the design of the study, to the drafting of the paper and performed the statistical analysis.

The authors report no conflict of interest in the present study.

The supplementary material for this article can be found at http://www.journals.cambridge.org/bjn

\section{References}

1. Lin H, Boylston TD, Chang MJ, et al. (1995) Survey of the conjugated linoleic acid contents of dairy products. J Dairy Sci 78, $2358-2365$.

2. Martin JC \& Valeille K (2002) Conjugated linoleic acids: all the same or to every one its own function? Reprod Nutr Dev 42, 525-536.

3. Pariza MW, Park Y \& Cook ME (2001) The biologically active isomers of conjugated linoleic acid. Progr Lipid Res 40, 283-298.

4. Riserus U, Vessby B, Arnlov J, et al. (2004) Effects of cis-9, trans-11 conjugated linoleic acid supplementation on insulin sensitivity, lipid peroxidation, and proinflammatory markers in obese men. Am J Clin Nutr 80, 279-283.

5. Clement L, Poirier H, Niot I, et al. (2002) Dietary trans-10, cis-12 conjugated linoleic acid induces hyperinsulinemia and fatty liver in the mouse. J Lipid Res 43, 1400-1409.

6. Riserus U, Smedman A, Basu S, et al. (2004) Metabolic effects of conjugated linoleic acid in humans: the Swedish experience. Am J Clin Nutr 79, 1146S-1148S.

7. Poirier H, Niot I, Clement L, et al. (2005) Development of conjugated linoleic acid (CLA)-mediated lipoatrophic syndrome in the mouse. Biochimie 87, 73-79.

8. Tsuboyama-Kasaoka N, Takahashi M, Tanemura $\mathrm{K}$, et al. (2000) Conjugated linoleic acid supplementation reduces adipose tissue by apoptosis and develops lipodystrophy in mice. Diabetes 49, 1534-1542.

9. Riserus U, Basu S, Jovinge S, et al. (2002) Supplementation with conjugated linoleic acid causes isomer-dependent oxidative stress and elevated C-reactive protein: a potential link to fatty acid-induced insulin resistance. Circulation 106, 1925-1929.

10. Ide T (2005) Interaction of fish oil and conjugated linoleic acid in affecting hepatic activity of lipogenic enzymes and gene expression in liver and adipose tissue. Diabetes 54, 412-423.

11. Liu LF, Purushotham A, Wendel AA, et al. (2007) Combined effects of rosiglitazone and conjugated linoleic acid on adiposity, insulin sensitivity, and hepatic steatosis in high-fat-fed mice. Am J Physiol Gastrointest Liver Physiol 292, G1671-G1682.

12. Bhattacharya A, Banu J, Rahman M, et al. (2006) Biological effects of conjugated linoleic acids in health and disease. J Nutr Biochem 17, 789-810.

13. de Deckere EA, van Amelsvoort JM, McNeill GP, et al. (1999) Effects of conjugated linoleic acid (CLA) isomers on lipid levels and peroxisome proliferation in the hamster. $\mathrm{Br} J$ Nutr 82, 309-317.

14. Macarulla MT, Fernández-Quintela A, Zabala A, et al. (2005) Effects of conjugated linoleic acid on liver composition and fatty acid oxidation are isomer-dependent in hamster. Nutrition 21, 212-219.

15. Gavrilova O, Haluzik M, Matsusue K, et al. (2003) Liver peroxisome proliferator-activated receptor $\gamma$ contributes to hepatic steatosis, triglyceride clearance, and regulation of body fat mass. J Biol Chem 278, 34268-34276. 
16. Diehl AM (2002) Nonalcoholic steatosis and steatohepatitis: IV. Nonalcoholic fatty liver disease abnormalities in macrophage function and cytokines. Am J Physiol 282, G1-G5.

17. Du H, Heur M, Duanmu M, et al. (2001) Lysosomal acid lipasedeficient mice. Depletion of white and brown fat, severe hepatosplenomegaly, and shortened life span. J Lipid Res 42, 489-500.

18. Reddy JK (2001) Nonalcoholic steatosis and steatohepatitis: III. Peroxisomal $\beta$-oxidation, PPAR $\alpha$, and steatohepatitis. Am $J$ Physiol 281, G1333-G1339.

19. Robertson G, Leclercq I \& Farrell GC (2001) Nonalcoholic steatosis and steatohepatitis: II. Cytochrome P-450 enzymes and oxidative stress. Am J Physiol 281, G1135-G1139.

20. Folch J, Lees M \& Sloane Stanley GH (1957) A simple method for the isolation and purification of total lipides from animal tissues. J Biol Chem 226, 497-509.

21. McGarry JD, Kuwajima M, Newgard CB, et al. (1987) From dietary glucose to liver glycogen: the full circle round. Annu Rev Nutr 7, 51-73.

22. Valeille K, Ferezou J, Amsler G, et al. (2005) A cis9,trans11conjugated linoleic acid rich-oil reduces the outcome of atherogenic process in hyperlipidemic hamster. Am J Physiol Heart Circ Physiol 289, H652-H659.

23. Simon P (2003) Q-Gene: processing quantitative real-time RTPCR data. Bioinformatics 19, 1439-1440.

24. Trygg J, Holmes E \& Lundstedt T (2007) Chemometrics in metabonomics. J Proteome Res 6, 469-479.

25. de Roos B, Rucklidge G, Reid M, et al. (2005) Divergent mechanisms of cis 9 ,trans 11 - and trans 10 , cis 12-conjugated linoleic acid affecting insulin resistance and inflammation in apolipoprotein $\mathrm{E}$ knockout mice: a proteomics approach. FASEB J 12, 1746-1748.

26. Trygg J \& Vold S (2002) Orthogonal projection to latent structure. J Chemometrics 16, 119-128.

27. Chavance M (1992) Jackknife and bootstrap. Rev Epidemiol Sante Publique 40, 209-218.

28. Caraux G \& Pinloche S (2005) Permutmatrix: a graphical environment to arrange gene expression profiles in optimal linear order. Bioinformatics 21, 1280-1281.

29. Nicholson JK, Holmes E, Lindon JC, et al. (2004) The challenges of modeling mammalian biocomplexity. Nat Biotechnol 22, $1268-1274$.
30. Degrace P, Demizieux L, Gresti J, et al. (2004) Hepatic steatosis is not due to impaired fatty acid oxidation capacities in C57BL/ $6 \mathrm{~J}$ mice fed the conjugated trans-10,cis-12-isomer of linoleic acid. J Nutr 134, 861-867.

31. Heilbronn LK \& Clifton PM (2002) C-reactive protein and coronary artery disease: influence of obesity, caloric restriction and weight loss. J Nutr Biochem 13, 316-321.

32. Poirier H, Shapiro JS, Kim RJ, et al. (2006) Nutritional supplementation with trans-10, cis-12-conjugated linoleic acid induces inflammation of white adipose tissue. Diabetes 55, 1634-1641.

33. Riserus U, Arner P, Brismar K, et al. (2002) Treatment with dietary trans 10 , cis 12 conjugated linoleic acid causes isomerspecific insulin resistance in obese men with the metabolic syndrome. Diabetes Care 25, 1516-1521.

34. Smedman A, Basu S, Jovinge S, et al. (2005) Conjugated linoleic acid increased C-reactive protein in human subjects. $\mathrm{Br} \mathrm{J}$ Nutr 94, 791-795.

35. Cortez-Pinto H, Zhi Lin H, Qi Yang S, et al. (1999) Lipids up-regulate uncoupling protein 2 expression in rat hepatocytes. Gastroenterology 116, 1184-1193.

36. Kohjima M, Enjoji M, Higuchi N, et al. (2007) Re-evaluation of fatty acid metabolism-related gene expression in nonalcoholic fatty liver disease. Int J Mol Med 20, 351-358.

37. LaRosa PC, Miner J, Xia Y, et al. (2006) Trans-10, cis-12 conjugated linoleic acid causes inflammation and delipidation of white adipose tissue in mice: a microarray and histological analysis. Physiol Genomics 27, 282-294.

38. Mitchell PL, Langille MA, Currie DL, et al. (2005) Effect of conjugated linoleic acid isomers on lipoproteins and atherosclerosis in the Syrian Golden hamster. Biochim Biophys Acta 1734, 269-276.

39. Nicolosi RJ, Rogers EJ, Kritchevsky D, et al. (1997) Dietary conjugated linoleic acid reduces plasma lipoproteins and early aortic atherosclerosis in hypercholesterolemic hamsters. Artery 22, 266-277.

40. Wilson TA, Nicolosi RJ, Chrysam M, et al. (2000) Conjugated linoleic acid reduces early aortic atherosclerosis greater than linoleic acid in hypercholesterolemic hamsters. Nutr Res 20, 1795-1805. 